\title{
Selective Arterial Embolisation of Uterine Arteriovenous Malformation in an Infertile Patient, subsequent Term Pregnancy and Postpartum Recurrence
}

\author{
Ercument Ciftci ${ }^{1}$, Ozgur Cakir ${ }^{1}$, Ozge Senem Yucel Cicek ${ }^{2}$ and Isa Cam ${ }^{1}$ \\ ${ }^{1}$ Department of Radiology, Faculty of Medicine, Kocaeli University, Kocaeli, Turkey \\ ${ }^{2}$ Department of Obstetrics and Gynaecology, Faculty of Medicine, Kocaeli University, Kocaeli, Turkey
}

\begin{abstract}
Uterine arteriovenous malformation (AVM) is a rare entity. This report describes selective catheterisation and embolisation of a uterine AVM in an infertile woman, subsequent spontaneous pregnancy and postpartum recurrence of the AVM treated with a second embolisation procedure. A 22-year woman presented with menometrorrhagia and failure to conceive. Pelvic magnetic resonance imaging showed a large uterine AVM. Selective catheterisation and embolisation of the AVM was performed. The patient conceived spontaneously two months later and delivered vaginally. A second embolisation was performed due to recurrence of uterine AVM at six months postpartum.

This is the first case reporting postpartum recurrence of a pre-conceptionally treated uterine AVM. Selective catheterisation and embolisation is a minimally-invasive, fertility-preserving procedure that successfully treats uterine AVM and should be the treatment of choice when the patient desires pregnancy. Successful pregnancy and vaginal delivery following embolisation is possible; however, an increased awareness of postpartum AVM recurrence is required.
\end{abstract}

Key Words: Fertility, Pregnancy, Therapeutic embolisation, Uterus, Arteriovenous malformation.

How to cite this article: Ciftci E, Cakir O, Cicek OSY, Cam I. Selective Arterial Embolisation of Uterine Arteriovenous Malformation in an Infertile Patient, subsequent Term Pregnancy and Postpartum Recurrence. J Coll Physicians Surg Pak 2022; 32(02):239-241.

\section{INTRODUCTION}

Uterine arteriovenous malformations (AVMs) are abnormal communications of arteries and veins by-passing capillaries. Although the true incidence of uterine AVM is unknown, it is a very rare condition, with less than 100 cases reported in the literature. ${ }^{1}$ One prospective study from Japan investigated the incidence of uterine AVM in women who had an abortion or delivery and reported this to be approximately $0.10 \%{ }^{2}$

Doppler ultrasound examination is the initial step for uterine AVM diagnosis. Subsequent magnetic resonance imaging (MRI) allows for accurate evaluation of the AVM. ${ }^{1}$ Since most women with uterine AVM are of reproductive age, the desire to maintain fertility influences the choice of available treatment options. Conservative management with embolisation treats uterine AVM and preserves the uterus. However, reports of successful pregnancy following this technique are scarce. ${ }^{3}$

Correspondence to: Dr. Ozge Senem Yucel Cicek, Department of Obstetrics and Gynaecology, Faculty of Medicine, Kocaeli University, Kocaeli, Turkey

E-mail: ozgesenemyucel@gmail.com

Received: December 16, 2019; Revised: February 27, 2020;

Accepted: March 09, 2020

DOI: https://doi.org/10.29271/jcpsp.2022.02.239
This case report describes selective arterial catheterisation and embolisation of a uterine AVM in a subfertile woman with subsequent spontaneous pregnancy leading to a live birth. Six months postpartum, there was a recurrence of the AVM which was successfully treated with a second embolisation procedure.

\section{CASE REPORT}

A 22-year woman, gravida 1, para 0 with a previous molar pregnancy, presented with heavy menometrorrhagia of eight months duration and failure to conceive. She had undergone uterine curettage for suspected mole one year prior to presentation and histopathological examination confirmed a diagnosis of complete hydatidiform mole (CHM). She had a history of referral to multiple clinics for vaginal bleeding and had been treated with estrogen and progesterone regimens. However, the bleeding was unresponsive to hormone therapy.

Color Doppler ultrasound examination showed a large, uterine AVM on the left side. Pelvic MRI revealed multiple, vascular-like, serpiginous structures within the myometrium (Figure $1 \mathrm{a}-\mathrm{c}$ ). As the patient desired future fertility, selective embolisation was planned. Patient's written informed consent for the procedure was obtained. Angiography revealed a $5 \times 3 \mathrm{~cm}$ AVM involving the left uterine artery (Figure 2a). Selective catheterisation of the left uterine artery was performed and the AVM was successfully occluded using 900-1200 $\mu \mathrm{m}$ and 700-900 $\mu \mathrm{m}$ acrylamido polyvinyl alcohol microspheres (a-PVAM) (BeadBlock; Biocom- 
patibles, Farnham, UK). Control angiography demonstrated complete occlusion of the uterine AVM with no residual flow through the arteriovenous shunt (Figure $2 b$ ). Her postoperative recovery was uneventful and she was discharged home on postoperative day 2 . The patient's menstrual cycles subsequently regularised and this was accompanied by decreased menstrual blood loss.
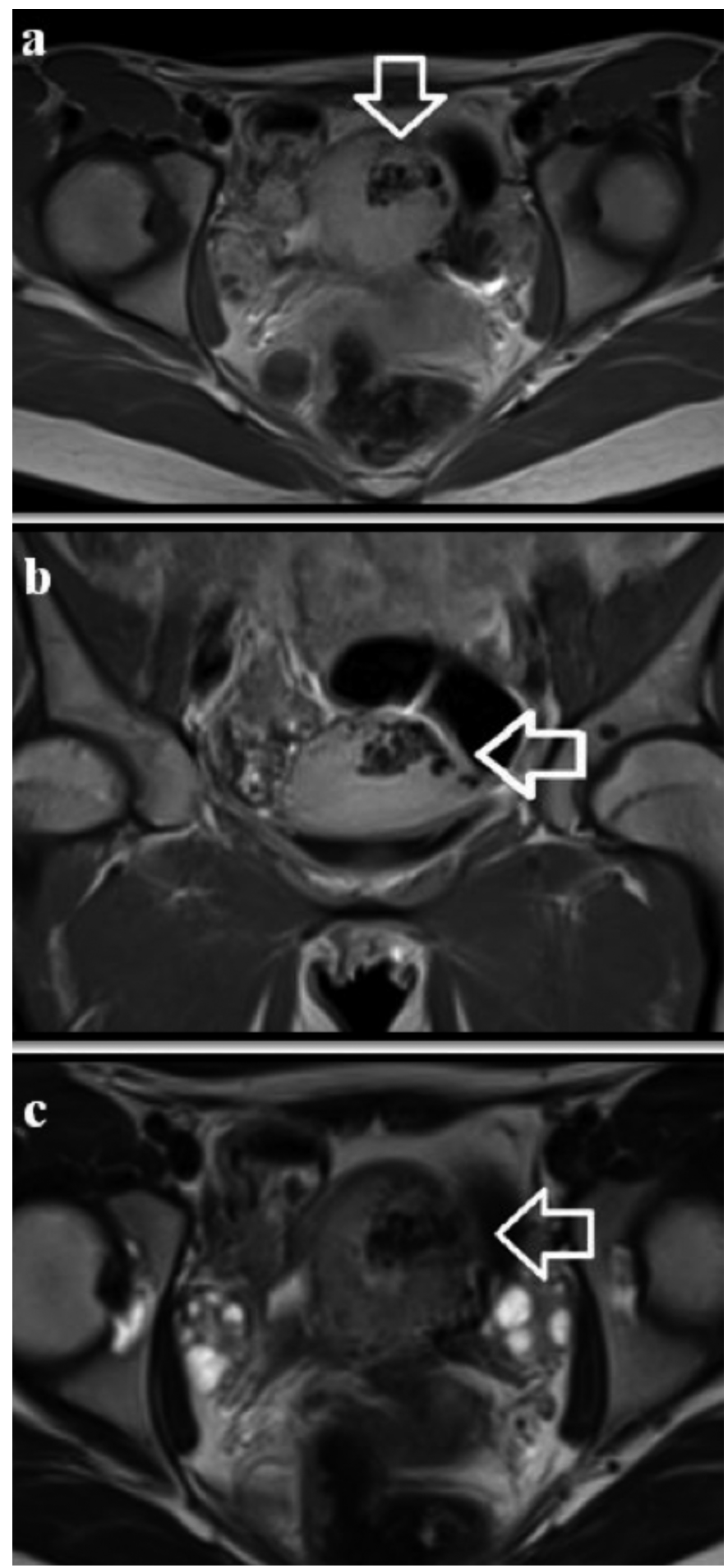

Figure 1: $(a, b, c)$ Pelvic MRI- (a) axial, (b) coronal, (c) post-contrast T1weighted sequences, T2-weighted TSE sequences. The images show multiple vascular-like serpiginious structures characterised by flow void in the lateral wall of the fundus (arrow).

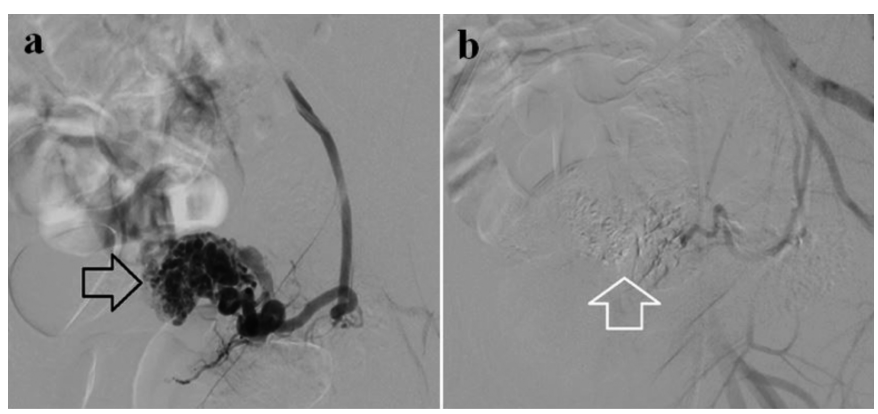

Figure 2: (a,b) Angiograms of first (a) pre-and (b) post-embolisation procedure. The pre-embolisation arteriogram showed a tangle of dilated vascular structures (black arrow), and post-embolisation images showed occluded vascularmalformation and embolising agent (white arrow).

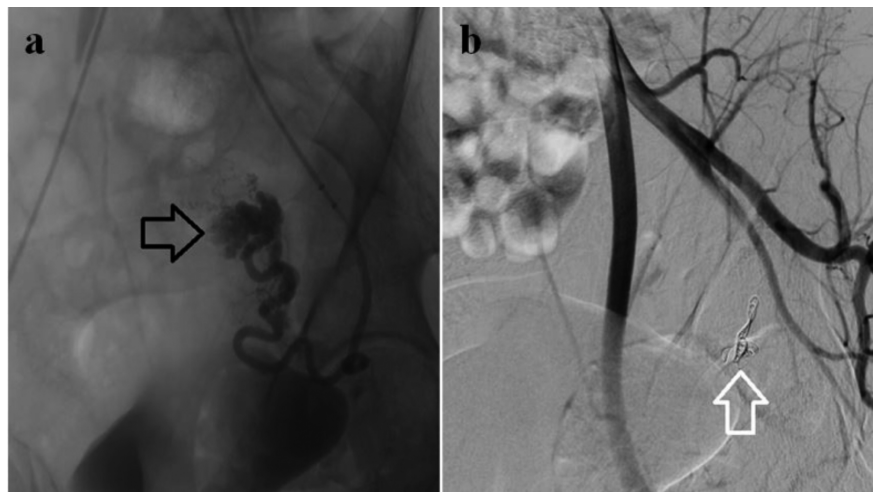

Figure 3: (a,b) Angiograms of the second (a) pre- and (b) post-embolisation procedure. The pre-embolisation arteriogram showed a tangle of vascular structures (black arrow), and post-embolisation images showed occluded left uterine iliac artery and vascular malformation with an embolisation coil (white arrow). Resolution of AVM is evident.

The patient conceivedspontaneously two months after the procedure. She had an uncomplicated pregnancy and labour began at 40 weeks. A healthy newborn, weighing 2,750 g with a 9-10-10 Apgar score, was delivered vaginally. Both the volume of postpartum bleeding and the postpartum recovery were normal. Six months after the birth, the patient presented to our emergency department with profuse vaginal bleeding and symptomatic anemia. Her hemoglobin concentration was $8.1 \mathrm{~g} / \mathrm{dL}$ and serum beta-human chorionic gonadotropin ( $\beta$-hCG) was negative. Color Doppler ultrasound revealed a $43 \times 28 \mathrm{~mm}$ myometrial lesion in left lateral wall of the uterus with hypoechoic areas demonstrating high velocity blood flow which indicated recurrence of the AVM. She received two units of red blood cells preoperatively. A second angiography, showing a tangle of dilated vessels, confirmed the presence of a recurrent AVM. Selective catheterisation of the left uterine artery was performed and AVM was occluded with 900-1200 $\mu \mathrm{m}$ a-PVAM followed by placement of a Target 360 Ultra $4 \mathrm{~mm} \times 10 \mathrm{~cm}$ coil (Stryker, Fremont, CA., USA). Post-embolisation angiography demonstrated complete embolisation of the AVM (Figure $3 a$ and $3 b$ ). Her postoperative hemoglobin concentration was $10.2 \mathrm{~g} / \mathrm{dL}$. She had an uneventful postoperative course and reported regular periods with light menstrual bleeding at six month follow-up.

\section{DISCUSSION}

Acquired AVM formation has been associated with uterine surgery and gestational trophoblastic disease. ${ }^{1,4}$ In this case, 
the patient experienced profuse vaginal bleeding after undergoing uterine evacuation for $\mathrm{CHM}$, suggesting that the molar pregnancy and/or uterine curettage may haveled directly to the acquired AVM.

Failure to conceive was the chief complaint together with vaginal bleeding in this case. In addition, the patient conceived spontaneously two months after embolisation. There are a few other reports of pregnancy shortly after embolisation of a uterine $\mathrm{AVM}{ }^{3}{ }^{3}$ Thus, it is plausible that the presence of a uterine AVM has a negative effect on fertility, although robust evidence for this causality is scarce.

There have only been a few previous reports of pregnancy following uterine AVM embolisation in the literature. ${ }^{3}$ Placental abnormalities and postpartum hemorrhage have been reported. ${ }^{5,6}$ Poor vascularisation of the treated area affecting placentation has been implicated as a potential cause. ${ }^{5}$ However, our case had an uneventful pregnancy course and an uncomplicated vaginal delivery. There are few other case reports describing successful pregnancy outcome. ${ }^{7}$ In the current case report, selective embolisation of the AVM and preservation of other arteries may have contributed to improve obstetric outcome. ${ }^{3}$

Recurrence of the AVM occurred at six months postpartum in our case. High recurrence rates up to $16.7 \%$ have been reported in the literature. ${ }^{8}$ However, this is the first case reporting postpartum recurrence of a pre-conceptionally treated uterine AVM. The pathogenesis of uterine AVMs has been associated with pregnancy and accompanying hormonal changes. ${ }^{6}$ Thus postpartum recurrence may be expected.

Clinicans should suspect a uterine AVM, especially in patients with unexplained persistent vaginal bleeding. Besides lifethreatening bleeding, uterine AVMs can also affect fertility. Selective catheterisation and embolisation is a minimally invasive, conservative alternative to hysterectomy and successfully treats uterine AVM. Successful pregnancy and vaginal delivery following embolisation is possible. However, clinicians should be aware of the recurrence of the AVM postpartum.

\section{ACKOWLEDGEMENT:}

The authors thank Mr Jeremy Jones of the Academic Writing Department of Kocaeli University, Izmit, Turkey, for his assistance in language editing and for his help and advice concerning the contents of this manuscript.

\section{PATIENT'S CONSENT:}

Written patient consent was obtained for publication of the case report.

\section{CONFLICT OF INTEREST:}

The authors declared no conflict of interest.

\section{AUTHORS' CONTRIBUTION:}

EC: Conception and design.

OC: Data collection, manuscript writing.

OSYC: Manuscript writing, submission, proofreading.

IC: Patient recruitment, data collection.

\section{REFERENCES}

1. Szpera-Gozdziewicz A, Gruca-Stryjak K, Breborowicz GH, Ropacka-Lesiak M. Uterine arteriovenous malformationdiagnosis and management. Ginekol Pol 2018; 89(5): 276-9. doi: 10.5603/GP.a2018.0047.

2. Yazawa H, Soeda S, Hiraiwa T, Takaiwa M, Hasegawa-Endo $\mathrm{S}$, Kojima $\mathrm{M}$, et al. Prospective evaluation of the incidence of uterine vascular malformations developing after abortion or delivery. J Minim Invasive Gynecol 2013; 20(3):360-7. doi: 10.1016/j.jmig.2012.12.008.

3. Delotte J, Chevallier P, Benoit B, Castillon JM, Bongain A. Pregnancy after embolisation therapy for uterine arteriovenous malformation. Fertil Steril 2006; 85(1):228. doi: 10.1016/j.fertnstert.2005.06.058.

4. Javed A, Chander R, Hoodbhoy Z. Uterine arteriovenous malformations after suction evacuation of missed miscarriage. J Coll Physicians Surg Pak 2018; 28(3):S33-S4. doi: 10.29271/jcpsp.2018.03.S33.

5. Soeda S, Kyozuka H, Suzuki S, Yasuda S, Nomura Y, Fujimori K. Uterine artery embolisation for uterine arteriovenous malformation is associated with placental abnormalities in the subsequent pregnancy: Two cases report. Fukushima J Med Sci 2014; 60(1):86-90. doi: 10.5387/fms.2013-13.

6. Wee A, Saxena R, Bagchi T. Secondary post-partum haemorrhage from a true uterine arteriovenous malformation. J Obstet Gynaecol 2018; 38(8):1169-70. doi: 10.1080/01443615.2017.1404017.

7. Ho CY, Seow KM, Huang LW, Tsai YL. Fertility outcomes following pelvic embolisation in women with acquired uterine arteriovenous malformation. Taiwan J Obstet Gynecol 2017; 56(6):831-5. doi: 10.1016/j.tjog.2017. 10.023.

8. Chen SQ, Jiang HY, Li JB, Fan L, Liu MJ, Yao SZ. Treatment of uterine arteriovenous malformation by myometrial lesion resection combined with artery occlusion under laparoscopy: A case report and literature review. Eur J Obstet Gynecol Reprod Biol 2013; 169(2):172-6. doi: 10.1016/j.ejogrb.2013.04.009. 